\title{
Functional Interactions Between Major Rice Blast Resistance Genes, $P i-t a$ and Pi-b, and Minor Blast Resistance Quantitative Trait Loci
}

\author{
Xinglong Chen, Yulin Jia, ${ }^{\dagger}$ Melissa H. Jia, Shannon R. M. Pinson, Xueyan Wang, and B. M. Wu
}

First and sixth authors: Department of Plant Pathology, China Agricultural University, Beijing, China 100193; and first, second, third, fourth, and fifth authors: U.S. Department of Agriculture-Agricultural Research Service (USDA-ARS), Dale Bumpers National Rice Research Center (DB NRRC), Stuttgart, AR 72160.

Accepted for publication 13 April 2018.

\begin{abstract}
Major blast resistance $(R)$ genes confer resistance in a gene-for-gene manner. However, little information is available on interactions between $R$ genes. In this study, interactions between two rice blast $R$ genes, $P i$ - $t a$ and $P i-b$, and other minor blast resistance quantitative trait loci (QTLs) were investigated in a recombinant inbred line (RIL) population comprising 243 RILs from a Cybonnet $($ CYBT) $\times$ Saber (SB) cross. CYBT has the $R$ gene $P i$-ta and SB has $P i-b$. Ten differential isolates of four Magnaporthe oryzae races (IB-1, IB-17, IB-49, and IE-1K) were used to evaluate disease reactions of the 243 RILs under greenhouse

conditions. Five resistance QTLs were mapped on chromosomes 2, 3, 8, 9 , and 12 with a linkage map of 179 single nucleotide polymorphism markers. Among them, $q B R 12(\mathrm{Q} 1)$, was mapped at the Pi-ta locus and accounted for $45.41 \%$ of phenotypic variation while $q B R 2(\mathrm{Q} 2)$ was located at the $P i-b$ locus and accounted for $24.81 \%$ of disease reactions. The additive-by-additive epistatic interaction between Q1 (Pi-ta) and Q2 $(P i-b)$ was detected; they can enhance the disease resistance by an additive 0.93 using the 0 to 9 standard phenotyping method. These results suggest that $P i-t a$ interacts synergistically with $P i-b$.
\end{abstract}

Rice blast caused by the fungus Magnaporthe oryzae is one of the most devastating crop diseases worldwide. M. oryzae is known to be highly adaptive to its hosts due to its hemibiotrophic nature. The crop losses due to blast would be enough to feed 60 million people annually (Pennisi 2010). Planting rice varieties with genetic resistance is the most efficient and economical way to reduce yield losses from blast disease although the disease is also controlled largely by fungicide application (Nalley et al. 2016). Classically, genetic resistance to $M$. oryzae has been identified with qualitative phenotyping data obtained under field conditions. To date, more than 100 major blast $R$ genes and 350 quantitative trait loci (QTLs) have been identified in rice germplasm (Ballini et al. 2008; Karamian et al. 2015; Sharma et al. 2012). Recently, studies have demonstrated that major $R$ genes and resistance QTLs can be identified reproducibly with blast races/isolates under greenhouse conditions (Jia and Liu 2011; Liu et al. 2015a, b; Ma et al. 2014; Wang et al. 2015; Yang et al. 2013). Major blast $R$ genes are often responsible for most of the resistance phenotypes and are only effective against those $M$. oryzae isolates containing the cognate avirulence ( $A V R)$ genes (Silué et al. 1992). However, the $A V R$ genes in $M$. oryzae are highly unstable, and resistance based on a single $R$ gene can be easily overcome shortly after its deployment, suggesting an urgent need to stack major blast $R$ genes for adequate and relatively durable resistance in varieties. Rice varieties with multiple resistance QTLs can enhance the resistance effect of major genes, and combining major $R$ genes and QTLs together will achieve more effective resistance to $M$. oryzae (Fukuoka et al. 2015). It is well documented that phenotypic effect of one gene often masks the effect of other $R$ genes (Carlborg and Haley 2004;

†Corresponding author: Y. Jia; E-mail: yulin.jia@ars.usda.gov

*The $\boldsymbol{e}$-Xtra logo stands for "electronic extra" and indicates that three supplementary figures and five supplementary tables are published online.

This article is in the public domain and not copyrightable. It may be freely reprinted with customary crediting of the source. The American Phytopathological Society, 2018.
Phillips 2008), and knowledge of nonallelic epistatic interaction of major $R$ genes and QTLs not only is important for understanding molecular mechanisms of blast resistance but also is essential for effective $R$ gene stacking. For example, Hittalmani et al. (2000) demonstrated that rice lines containing two to three of the $R$ genes, $P i-1, P i-z 5$, and $P i$-ta, were more resistant to blast disease than each of the $R$ genes individually. Similarly, more enhanced resistance was observed in rice lines containing $\mathrm{Pi}-1, \mathrm{Pi}$-2, and $\mathrm{Pi}$ 33 or with $\mathrm{Pi}-2$ and $\mathrm{Pi}-54$ than in lines containing a single $R$ gene (Chen et al. 2008; Ratna Madhavi et al. 2016). Enhanced resistance was also observed when Pi46(t) and Pi-ta were stacked together (Xiao et al. 2016).

$P i-b$ and $P i$-ta were the first two blast $R$ genes cloned and both of them encode nucleotide-binding and leucine-rich repeat (NLR) domains predicted to be cytoplasmic proteins (Bryan et al. 2000; Wang et al. 1999). Subsequent intensive studies of these two $R$ genes led to a better understanding of the molecular basis of disease resistance and improved genetic markers that have been implemented in breeding programs worldwide to monitor their resistant functions (Jia et al. 2000, 2002; 2003a, b, 2004a, b; Lee et al. 2009; Wang et al. 2001). The U.S. tropical japonica rice variety Cybonnet (CYBT) (PI 636726) is a long-grain, semidwarf variety with good yield potential, blast resistance, and excellent milling quality. CYBT has Pi-ta and Pi-km (Gibbons et al. 2006) and its response to different blast races including avirulent isolates of IB-1, IB-17, and IB-49 and the virulent isolate TM2 of IE-1K has been characterized (Fjellstrom et al. 2004). Saber (SB) (PI 633624) is an early-maturing, long-grain cultivar with improved blast resistance. SB has $P i-b$ that confers resistance to IB-1, IB-17, and IE-1K and is partially resistant to IB-49 (McClung et al. 2004). A set of CYBT $\times$ SB recombinant inbred lines (CS-RILs) was successfully used to map QTLs for resistance to kernel fissuring (Pinson et al. 2013).

The objectives of this study were to (i) determine the phenotypic effects of $P i$-ta and $P i-b$, and other minor blast resistance QTLs according to the responses of the 243 RILs to 10 commonly found blast isolates belonging to four races under greenhouse conditions; (ii) examine epistatic interactions between $\mathrm{Pi}$-ta and $\mathrm{Pi}$ - $b$ 
and other QTLs; and (iii) develop rice genetic stocks with single nucleotide polymorphism (SNP) marker-associated $R$ genes for marker-assisted breeding.

\section{MATERIALS AND METHODS}

Plant materials and growth. A total of 277 CS-RILs was created using single-seed descent in the greenhouse at USDA-ARS, Dale Bumpers National Rice Research Center (DB NRRC), Stuttgart, Arkansas (Y. Jia, unpublished data). After removing nonparental and skewed segregated lines, $243 \mathrm{~F}_{9}$ RILs were selected for QTL mapping. Five to seven seeds were sowed into a black plastic insert $(2.8 \times 3.8 \times 3.8 \mathrm{~cm}$ length, width, and depth $)$ with a hole at the bottom, a total of 96 seeded inserts containing steam sterilized clay soil from the local field were placed in a tray $(25.4 \times 50.8 \mathrm{~cm})$. Seedlings were grown at 24 to $30^{\circ} \mathrm{C}$ with an $8 \mathrm{~h}$ dark and $16 \mathrm{~h}$ light cycle in the greenhouse. After germination, about 50 to $100 \mathrm{mg}$ of controlled-release fertilizer (Osmocote Plus, 15-9-12 [N-P-K]; Hummert International, Earth City, MO) was added atop each insert. Inserts were thinned at the V2 to V3 stage to two to three uniform seedlings and grown to the V3 to V4 leaf stage (approximately 18 days after sowing) (Counce et al. 2000) before blast inoculation or DNA preparation.

Races (isolates) of $M$. oryzae and spore production. Ten $P i-k m$ virulent isolates of four races of $M$. oryzae, IB-1 (12A23, 15A6, 15L22), IB-17 (14L42, 88A110, SSID10), IB-49 (ZN52, ZN61, ML1), and IE-1K (TM2), were selected to exclude the effect of $\mathrm{Pi}-\mathrm{km}$ (Supplementary Fig. S1). Desiccated filter paper pieces containing mycelia and spores were removed from the $-20^{\circ} \mathrm{C}$ freezer, and placed on oatmeal agar plates (BD Difco, Franklin Lakes, NJ) under black-white fluorescent light for 7 to 10 days at 23 to $25^{\circ} \mathrm{C}$. Spores were harvested with $0.25 \%$ gelatin (Fisher Scientific, Waltham, MA) solution and filtered through four layers of cheesecloth. The concentration was adjusted to 0.3 to $9 \times 10^{5}$ spores $\mathrm{ml}^{-1}$ with a hemocytometer according to the manufacturer's instruction.

Experimental design. To study the interaction between $\mathrm{Pi}$-ta and $P i-b$, the $\mathrm{F}_{9} \mathrm{CYBT} \times \mathrm{SB}$ population, characterized by SNP markers, was used for blast disease evaluation and QTL mapping. Ten $P i-k m$ virulent isolates of four races of $M$. oryzae were selected to inoculate this population, including the parental lines (Table 1). Rice cultivar M-202 was added as a positive control. Seedlings were randomly placed in the trays for inoculation and each treatment was replicated three to eight times under greenhouse conditions. A categorical rating method was used to determine the disease reactions. The interaction between $P i$-ta and $P i-b$ was evaluated through QTL mapping.

Blast inoculation, evaluation, and statistical analysis. A tray of rice seedlings (160 to 288 CS-RILs) was placed in a black plastic bag and was evenly sprayed with 30 - to $45-\mathrm{ml}$ spore suspensions $\left(0.3\right.$ to $9 \times 10^{5}$ spores $\left.\mathrm{ml}^{-1}\right)$ using an artist airbrush (Badger Air-Brush Co., Franklin Park, IL). The inoculated bags were sealed immediately to maintain relative humidity at 90 to $100 \%$ for $24 \mathrm{~h}$ and then transferred to a fungal incubation room in the greenhouse which maintained an environment with $80 \%$ relative humidity at $24^{\circ} \mathrm{C}$ with an $8 \mathrm{~h}$ dark and $16 \mathrm{~h}$ light cycle. Disease evaluation was performed on the seventh day postinoculation at the second youngest leaf by the standard visual scale (0 to 9 ) based on a system developed by the International Rice Research Institute (IRRI 2002) (Supplementary Fig. S2) where $0=$ no lesions visible; $1=$ small pinpoint or slightly larger brown speck without an open or sporulating center; $2=$ small round to oval spots, about 1 to $2 \mathrm{~mm}$ in diameter, with gray or necrotic centers with brown margins; 3 = lesion type is similar to lesions described in scale 2 , but with many more lesions on the upper leaves; $4=$ lesions type is similar to lesions described in scale 3 (typical susceptible blast lesions) but is $3 \mathrm{~mm}$ or longer, and less than $4 \%$ of the leaf area is infected; $5=4$ to $10 \%$ of the leaf area is infected with typical blast lesions; $6=11$ to $25 \%$ of the leaf area is infected with typical blast lesions; $7=$ typical blast lesions infect 26 to $50 \%$ of the leaf area; $8=51$ to $75 \%$ of the leaf area is infected with typical blast lesions with many dead leaves; and $9=$ more than $75 \%$ of the leaf area is affected or dead. All the experiments were performed in the greenhouse at DB NRRC from January 2016 to May 2017. The averages and ranges of the ratings were calculated for each RIL in Microsoft Excel 2010. Analysis of variance (GLM) was performed to analyze the effects of parental disease reaction in SAS (Version 9.2, SAS Institute Inc., Cary, NC).

Genomic DNA extraction and SNP markers. Genomic DNA was extracted using the Qiagen Dneasy Plant Mini Kit (Qiagen, Valencia, CA). Two hundred and fifty nanograms of each sample was assayed with the Rice OPA 4.0 (Thomson et al. 2012) 384-SNP Illumina Golden Gate oligo pool assay (Illumina Inc., San Diego, CA) using the BeadXpress platform, according to the manufacturer's protocol. Allele calls were performed using the genotyping module of Genome Studio v2011.1 (Illumina Inc.). We manually checked SNP-call in Genome Studio for quality and accuracy because rice is inbred, but the SNP detection algorithm developed by Genome Studio was originally for out-crossing and default setting is for three SNP markers (two homozygotes and one heterozygote).

Linkage map and QTL analysis. The linkage map for the $\mathrm{F}_{8}$ CS-RILs was constructed using 179 polymorphic SNP markers and R statistical software (R Core Team 2013) with the qtl package (Broman and Sen 2009). The SNP markers were assigned to linkage groups based on a maximum recombination fraction and a minimum logarithm of odds (LOD) score. The genetic distances and the confidence intervals were calculated by Kosambi map function (Kosambi 1943) and 95\% Bayesian credibility interval method, respectively (Sen and Churchill 2001). QTLs were scanned by Haley-Knott regression with 1 centimorgan (cM) and implemented for the fit of a multiple-QTL model. To estimate the genome-wide significance threshold, all genotyping data were applied to perform the permutation test for 1,000 times at a significance level of 0.05 (Churchill and Doerge 1994). The QTL effects were evaluated by fit qtl function, the statistical formula $=\mathrm{y} \sim \mathrm{Q} 1+\mathrm{Q} 2+\mathrm{Q} 1 * \mathrm{Q} 2, \mathrm{y} \sim \mathrm{Q} 1+\mathrm{Q} 2+\mathrm{Q} 3+\mathrm{Q} 4+\mathrm{Q} 1 * \mathrm{Q} 2$. Effect plot function was used to detect the possible epistatic interactions between QTLs/markers (Broman and Sen 2009). Putative QTLs were named according to the linkage groups. The genetic map was visualized using MapChart, version 2.2 (Voorrips 2002).

TABLE 1. Disease reactions of the parental rice cultivars Cybonnet and Saber to 10 isolates (four races) of Magnaporthe oryzae

\begin{tabular}{|c|c|c|c|c|c|c|c|c|c|c|c|}
\hline \multirow[b]{2}{*}{ Isolates } & \multicolumn{3}{|c|}{ IB-1 } & \multicolumn{3}{|c|}{ IB-17 } & \multicolumn{3}{|c|}{ IB-49 } & \multirow[b]{2}{*}{ Mean } & \multirow{2}{*}{$\frac{\text { IE-IK }}{\text { TM2 }}$} \\
\hline & $12 \mathrm{~A} 23^{\mathrm{w}}$ & $15 \mathrm{~A} 6^{\mathrm{w}}$ & $15 \mathrm{~L} 22^{\mathrm{w}}$ & $14 \mathrm{~L} 42^{w}$ & $88 \mathrm{~A} 110^{\mathrm{w}}$ & SSID10w & ZN52x & ZN61 ${ }^{x}$ & ML1 $^{y}$ & & \\
\hline Cybonnet & 0.67 & 3.00 & 2.80 & 0.00 & 1.00 & 0.50 & 0.75 & 0.80 & 0.25 & $1.11 \mathrm{~b}^{\mathrm{z}}$ & 6.00 \\
\hline Saber & 4.00 & 3.33 & 3.00 & 1.30 & 1.33 & 1.20 & 2.00 & 1.60 & 0.75 & $1.95 \mathrm{a}$ & 3.00 \\
\hline
\end{tabular}

w Isolates described by Wang et al. (2017b).

x Isolates described by Correll et al. (2000).

y ML1 indicates an isolate purified from race IB-49.

z Mean was calculated with all the isolates except TM2 by GLM in SAS. Different letters indicate that the means are significantly different $(P<0.05)$. 


\section{RESULTS}

SNP markers and linkage groups. Two hundred and thirteen polymorphic SNP markers were initially used to genotype the CYBT $\times$ SB population; however, 34 markers were excluded due to high heterogeneity or high levels of missing data. These linkage groups were mapped on 12 chromosomes, covering $1334.42 \mathrm{cM}$ with an average genetic distance of $7.45 \mathrm{cM}(0.13$ to $45.29 \mathrm{cM}$ ) between adjacent markers. Chromosome 1 was divided into two linkage groups because the interval between marker rd1000152 and id 1017334 was larger than $50 \mathrm{cM}$ (Supplementary Fig. S3).

Disease reactions of $P i$ - $t a$ and $P i-b$ containing parents and their RILs. $P i$-ta containing rice cultivars were resistant to all isolates of races IB-1, IB-17, and IB-49, except IE-1K (TM2). As expected, CYBT carrying $P i$ - $t a$ was susceptible to IE-1K with a mean rating of 6.00 ; conversely, SB, carrying $P i-b$, was resistant with a mean rating of 3.00 on the 0 to 9 scale phenotyping methods (Table 1). CYBT was more resistant to all isolates, except TM2, with a mean of 1.11 (0 to 3.00) compared with SB with mean ratings of $1.95(0.75$ to 4.00$)$ significantly $(P=0.01)$ (Table 1$)$. Frequency distributions of disease reactions of all the CS-RILs with their parental lines to each isolate are shown in Figure 1 (Supplementary Table S1). There were some RILs that were more resistant or more susceptible than the parents suggesting that transgressive segregation occurred for blast reactions. Genetic analysis revealed that two major R/QTLs are responsible for resistance to all isolates except for TM2. Only one major $R / \mathrm{QTL}$ was identified to be responsible for resistance to TM2 (Supplementary Table S2). As expected, most of the RILs (approximately 77.82\%) were resistant to all the isolates except for TM2. As shown in Table 2, there were 53 lines that contain no major $R$ gene ( $P i$-ta or $P i-b)$ with a different combination of minor QTLs, as expected all the lines were susceptible (mean $\geq 5$ ) to all the isolates except one line was resistant with a mean of 1.11. There were 69 lines that contain $P i$-ta, but no $P i$ - $b$, with a different combination of minor QTLs, all the lines were resistant to all of the isolates except TM2. There were 60 lines that contained $P i-b$, but no $P i$-ta, with a different combination of minor QTLs, all the lines were resistant to nine isolates except one line showed susceptible with a mean of 6.45. There were 59 lines with both major $R$ genes ( $P i$-ta and $P i-b$ ) with a different combination of minor QTLs, all the lines were resistant to nine isolates. As for the virulent isolate TM2, there were 122 lines without $P i$ - $b$ but with minor QTLs, almost all these lines were susceptible. On the other hand, 119 lines contain $P i-b$ and different minor QTLs, most lines showed resistance while five lines were susceptible (Table 2). Taken together, these data suggest that the majority of phenotype variation observed in this population was due to $P i$-ta and $P i-b$.

Functional assignment of Pi-ta (Q1) and Pi-b (Q2) in different genetic backgrounds. A total of five resistance QTLS were mapped with 10 isolates (four races) of $M$. oryzae, two of which were from CYBT and three from SB (Table 3; Fig. 2). There were two major resistance QTLs. The first one named Q1 (qBR12), $P i$-ta, was mapped to the Pi-ta locus between 10.60 and 10.86 megabases $(\mathrm{Mb})$ on chromosome 12 near SNP markers FNP_2 and id12004228, was detected by all isolates, except isolate TM2, with a mean LOD of 44.41 (32.50 to 55.24) and it accounted for $45.41 \%$ (33.79 to $53.46 \%$ ) of the phenotypic variation (Table 3; Supplementary Table S3). The second, Q2 (qBR2), Pi-b, was located at the last SNP marker id2015934 (35.03 Mb) on chromosome 2. It was mapped with a mean LOD of 28.33 (20.71 to 32.86) and accounted for $24.81 \%$ (16.85 to $33.14 \%$ ) of the disease reaction; as for isolate TM2, Q2 (Pib) was found with a LOD of 80.74 and accounted for $78.37 \%$ of the phenotypic variation (Table 3 ).

TABLE 2. Summary of disease reactions of recombinant inbred lines (RILs) carrying indicated major blast $R /$ quantitative trait loci ${ }^{\mathrm{x}}$

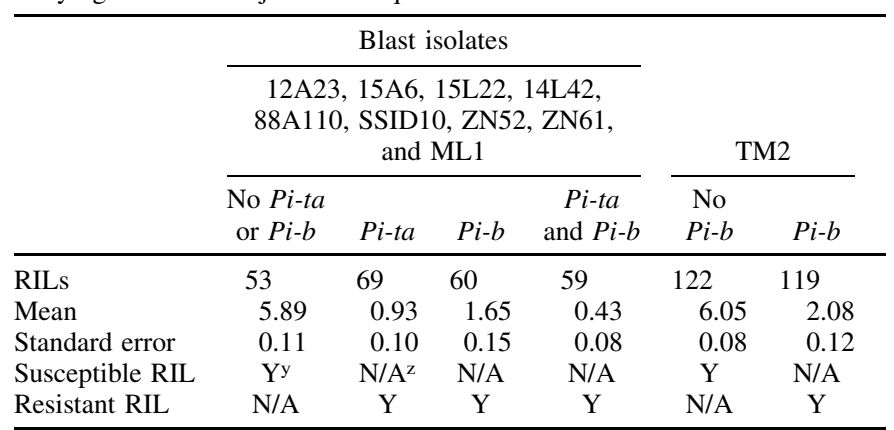

${ }^{\mathrm{x}}$ Disease reactions were evaluated on the 0 to 9 rating scale, where 0 to 4 is resistant and 5 to 9 is susceptible.

y Y denotes yes for the corresponding disease reaction.

${ }^{\mathrm{z}}$ N/A denotes not applied.

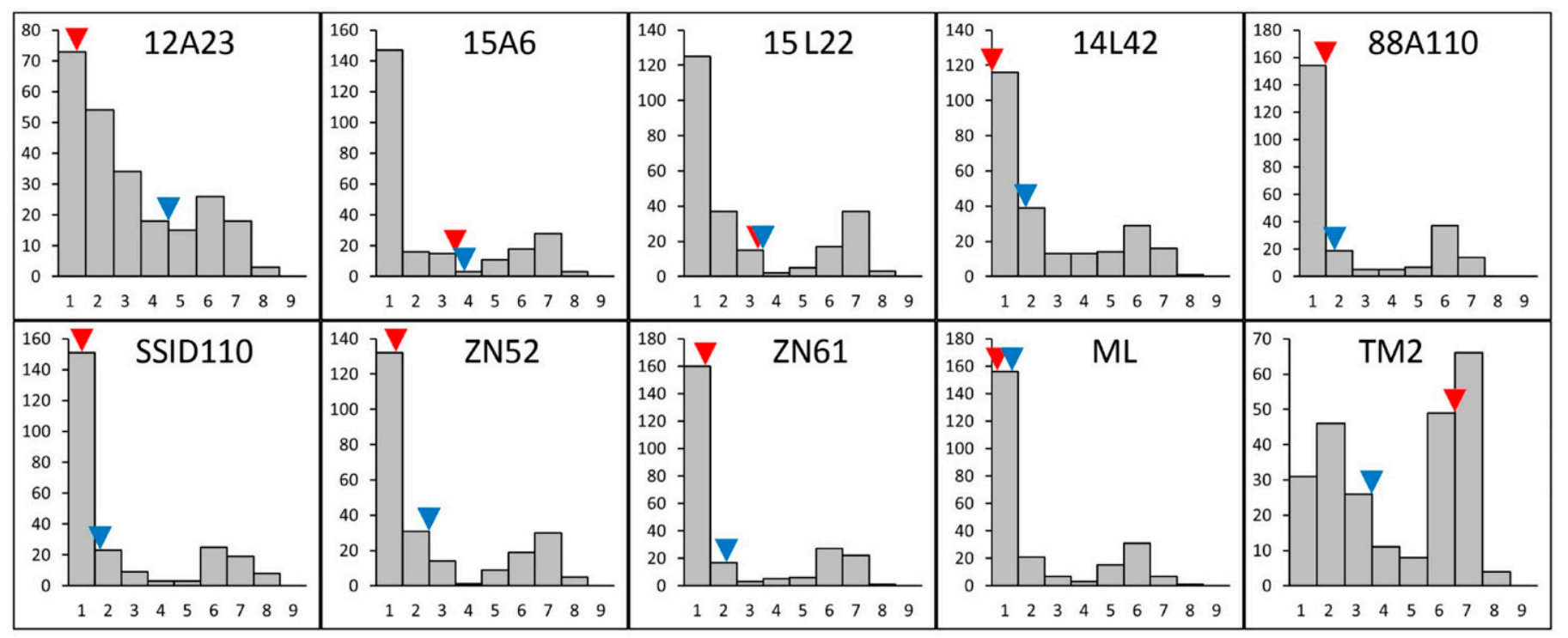

Fig. 1. Frequency distribution of disease reactions of all the recombinant inbred lines (RILs; 243 lines) to each isolate of Magnaporthe oryzae. Light gray (red) and dark gray (blue) arrows indicate the disease score of parental CYBT and SB to each isolate, respectively. $\mathrm{x}$-axis indicates disease reaction, and $\mathrm{y}$-axis indicates the number of RILs. 
TABLE 3. Locations, effects, and contributions of resistance quantitative trait loci (QTLs) in CYBT $\times$ SB population ${ }^{\mathrm{u}}$

\begin{tabular}{|c|c|c|c|c|c|c|c|c|}
\hline QTLs & Chr. & M. oryzae isolate & Marker interval & Nearest marker locus ${ }^{\mathrm{v}}$ & LOD value & $\operatorname{PVE}(\%)^{\mathrm{w}}$ & $\mathrm{AE}(\mathrm{est})^{\mathrm{x}}$ & Reference $^{y}$ \\
\hline Q1(qBR12) & 12 & $12 \mathrm{~A} 23$ & id12003803-rd12003441 & id12004228 (10.86) & 47.01 & 47.55 & 1.49 & Hittalmani et al. 2000 \\
\hline Q2(qBR2) & 2 & $12 \mathrm{~A} 23$ & id2015653-id2015934 & id2015934 (35.03) & 30.34 & 25.67 & -1.09 & Wang et al. 1999 \\
\hline Q1(qBR12) & 12 & $15 \mathrm{~A} 6$ & id12003803-rd12003441 & FNP_2 (10.60) & 32.50 & 33.79 & 1.45 & Hittalmani et al. 2000 \\
\hline Q2(qBR2) & 2 & $15 \mathrm{~A} 6$ & id2015653-id2015934 & id2015934 (35.03) & 32.04 & 33.14 & -1.43 & Wang et al. 1999 \\
\hline Q1(qBR12) & 12 & $15 \mathrm{~L} 22$ & FNP_2-rd12003441 & id12004228 (10.86) & 55.24 & 49.80 & 1.80 & Hittalmani et al. 2000 \\
\hline Q2(qBR2) & 2 & $15 \mathrm{~L} 22$ & id2015653-id2015934 & id2015934 (35.03) & 26.71 & 17.70 & -1.07 & Wang et al. 1999 \\
\hline Q3(qBR8) & 8 & $15 \mathrm{~L} 22$ & rd8002298-id8002877 & $\operatorname{rd} 8002343(7.61)$ & 3.98 & 2.10 & 0.56 & Xing et al. 2015 \\
\hline Q4(qBR9) & 9 & $15 \mathrm{~L} 22$ & id9006947-rd9002766 & id9007173 (20.78) & 3.57 & 1.88 & -0.36 & Jia and Liu 2011 \\
\hline Q1(qBR12) & 12 & $14 \mathrm{~L} 42$ & id12003803-rd12003441 & id12004228 (10.86) & 48.79 & 53.46 & 1.64 & Hittalmani et al. 2000 \\
\hline Q2(qBR2) & 2 & 14L42 & id2015653-id2015934 & id2015934 (35.03) & 20.71 & 16.85 & -0.92 & Wang et al. 1999 \\
\hline Q1(qBR12) & 12 & $88 \mathrm{~A} 110$ & id12003803-rd12003441 & id12004228 (10.86) & 39.22 & 41.25 & 1.46 & Hittalmani et al. 2000 \\
\hline Q2(qBR2) & 2 & 88A110 & id2015653-id2015934 & id2015934 (35.03) & 29.08 & 27.48 & -1.19 & Wang et al. 1999 \\
\hline Q1(qBR12) & 12 & SSID10 & id12003803-rd12003441 & id12004228 (10.86) & 42.08 & 46.06 & 1.68 & Hittalmani et al. 2000 \\
\hline Q2(qBR2) & 2 & SSID10 & id2015653-id2015934 & id2015934 (35.03) & 24.26 & 22.00 & -1.16 & Wang et al. 1999 \\
\hline Q1(qBR12) & 12 & ZN57 & FNP_2-rd12003441 & id12004228 (10.86) & 52.69 & 53.15 & 1.87 & Hittalmani et al. 2000 \\
\hline Q2(qBR2) & 2 & ZN57 & id2015653-id2015934 & id2015934 (35.03) & 28.33 & 21.98 & -1.20 & Wang et al. 1999 \\
\hline Q1(qBR12) & 12 & ZN61 & id12003803-rd12003441 & id12004228 (10.86) & 38.91 & 40.27 & 1.52 & Hittalmani et al. 2000 \\
\hline Q2(qBR2) & 2 & ZN61 & id2015653-id2015934 & id2015934 (35.03) & 30.62 & 29.02 & -1.29 & Wang et al. 1999 \\
\hline Q1(qBR12) & 12 & $\mathrm{ML}^{\mathrm{z}}$ & id12003803-rd12003441 & FNP_2 (10.60) & 43.22 & 43.33 & 1.46 & Hittalmani et al. 2000 \\
\hline Q2(qBR2) & 2 & $\mathrm{ML1}^{\mathrm{z}}$ & id2015653-id2015934 & id2015934 (35.03) & 32.86 & 29.49 & -1.20 & Wang et al. 1999 \\
\hline Q2(qBR2) & 2 & TM2 & id2015934-id2015934 & id2015934 (35.03) & 80.74 & 78.37 & -2.03 & Wang et al. 1999 \\
\hline Q5(qBR3) & 3 & TM2 & id3010971-id3014633 & id3013770 (29.25) & 3.05 & 1.28 & -0.27 & Wu et al. 2016 \\
\hline
\end{tabular}

u A total of 10 isolates was used to inoculate CS-RIL population, and the average of three to eight measurements was used for QTL mapping.

$\checkmark$ Numbers in brackets represent the physical locations (MB) of the nearest markers on rice chromosomes.

${ }^{w}$ Phenotypic variation explained by an individual QTL.

$x$ Estimated additive effects.

y Indicate these QTLs were also identified in other studies.

z ML1 indicates an isolate purified from race IB-49.

\section{CHR2 \\ CHR3 \\ CHR8 \\ CHR9 \\ CHR12}

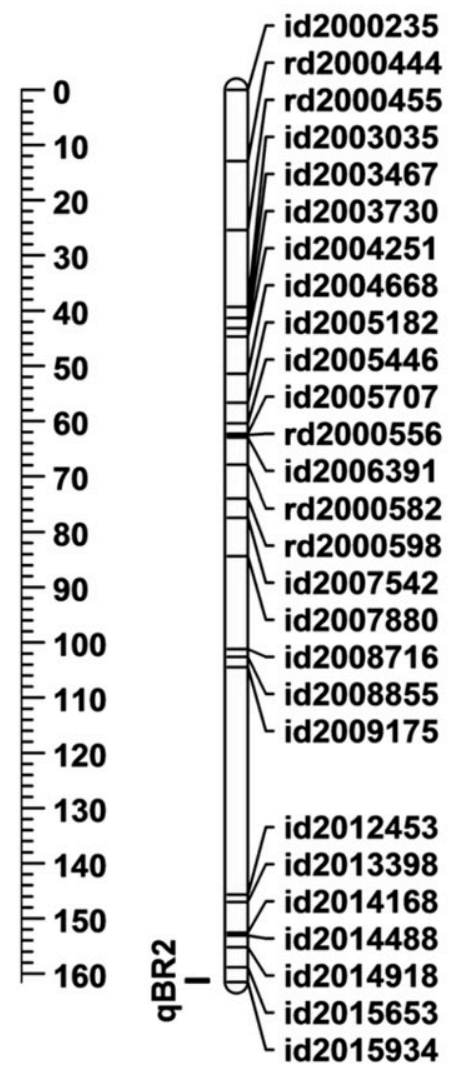

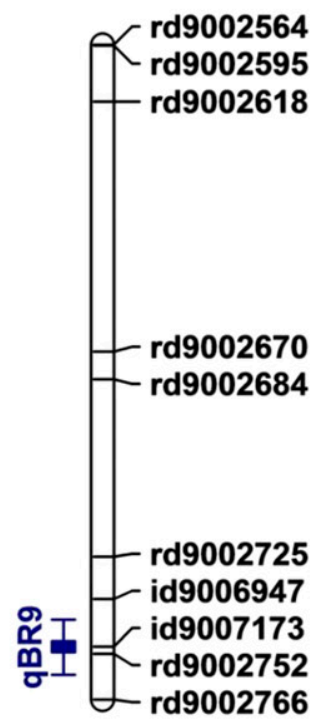

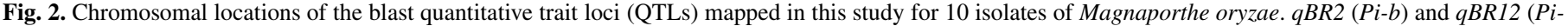
ta) were mapped by isolate $12 \mathrm{~A} 23$ as two representative QTLs of all the isolates. 
The remaining three QTLs of smaller individual effect were located on chromosomes 3, 8, and 9. By using the isolate 15L22, two resistance QTLs, Q3 ( $q B R 8)$ and Q4 ( $q B R 9)$, were detected between SNP markers rd8002298 and id8002877 on chromosome 8 and between id9006947 and rd9002766 on chromosome 9 with LODs of 3.98 and 3.57 , accounting for 2.10 and $1.88 \%$ of the phenotypic variation, respectively (Table 3). Q5 (qBR3) was identified by using isolate TM2 (IE-1K) between id3010971 and id3014633 with a LOD of 3.05 that accounted for $1.28 \%$ of phenotypic variation (Table 3).

Additive-by-additive epistatic interaction of Pi-ta, Pi-b, and other QTLs. The mean additive effects of Q1 (Pi-ta) and Q2
(Pi-b) were 1.60 (1.45 to 1.87 ) and -1.17 (-1.43 to -0.92$)$, except for isolate TM2 using the 0 to 9 phenotyping method, indicating that $P i-t a$ and $P i-b$ may reduce the lesion scale by 1.60 and 1.17 , respectively (Fig. 3). Epistatic effect between these two major genes was consistently detected by using all of the isolates except TM2 in R/QTL (Fig. 4), and estimated to be significant $(P<0.001)$ by the formula $\mathrm{y} \sim \mathrm{Q} 1+\mathrm{Q} 2+\mathrm{Q} 1 * \mathrm{Q} 2, \mathrm{y} \sim \mathrm{Q} 1+\mathrm{Q} 2+\mathrm{Q} 3+\mathrm{Q} 4+\mathrm{Q} 1 * \mathrm{Q} 2$. The result showed that a strong epistatic effect came from the same direction as $P i-b$ (contributed by SB alleles), and varied from each isolate excluding TM2, with a mean effect of -0.93 ( -1.15 to -0.6$)$, suggesting that they can decrease the disease by an additional 0.93 (Figs. 3 and 4). This additive to additive epistatic interaction can
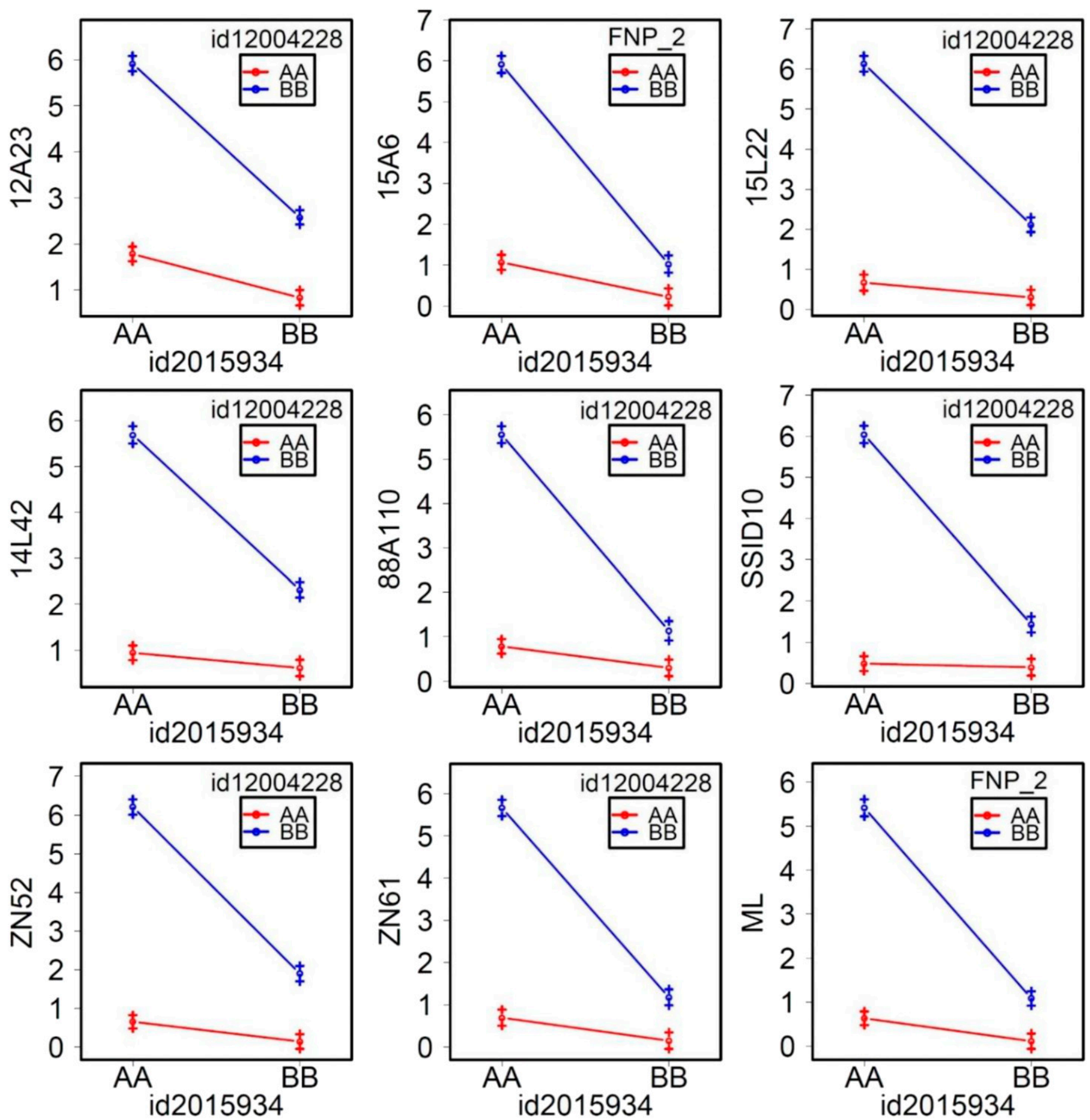

Fig. 3. Epistatic interaction plot between Pi-ta (marker FNP_2, id12004228) and Pi-b (marker id2015934). y-axis indicates disease reaction rating. 
account for an additional $15.22 \%$ (7.66 to 21.13 ) of the phenotypic variation for the disease resistance in this population (Supplementary Table $\mathrm{S} 4$ ).

As for TM2, the additive effect of $P i b$ was -2.03 and accounted for $78.37 \%$ of phenotypic variation explained (Table 3; Supplementary Table S5). To examine if Pi-ta influences the outcome of disease reactions of RILs with a different combination of minor QTLs, we compared the average disease reaction of $\mathrm{Pi}$-ta containing RILs and $P i-b$ containing RILs after inoculation with $P i$ - $t a$ virulent isolate and $P i$ - $b$ avirulent isolate, TM2. As shown in Figure 5, in the compatible interaction there was no difference in disease reactions of RILs with and without Q1 (Pi-ta). However, differences of disease reactions were observed for RILs with and without Q2 $(P i-b)$ in the compatible interaction validating the added effect of interactions of $P i-b$ with other minor QTLs.

Epistatic interactions between major and minor QTLs were also detected significantly $(P<0.05)$ by using isolate $15 \mathrm{~L} 22$. Their epistatic effects were $-1.18,0.22,0.24$ between Q1 (Pi-ta) and Q4

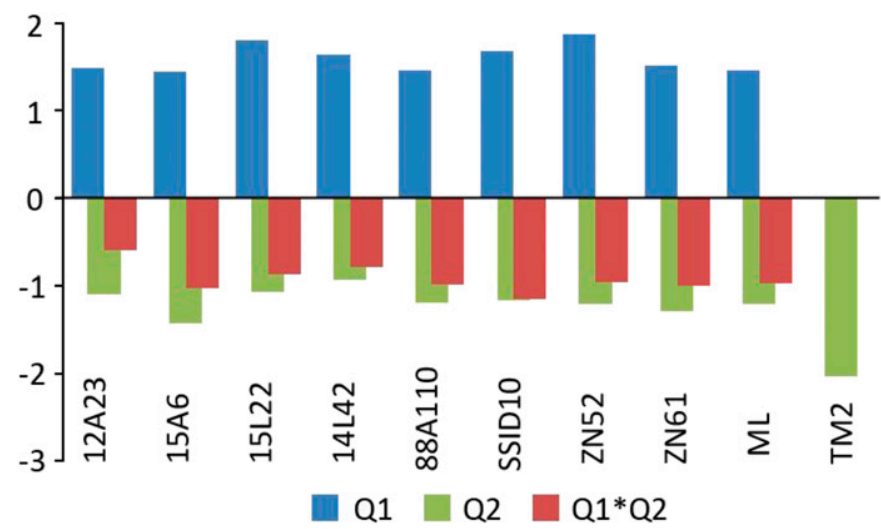

Fig. 4. The additive effect and their epistatic effect of the major resistant quantitative trait locus (QTL). Q1 denotes $P i-t a, \mathrm{Q} 2$ denotes $P i-b$, and Q1*Q2 denotes their epistatic effect. $y$-axis positive and negative indicates the effect contributed by the alleles of CYBT and SB, respectively.
(qBR9); Q2 (Pi-b) and Q4 (qBR9); Q1 (Pi-ta), Q2 (Pi-b), and Q3 (qBR8); and accounted for $0.48,0.70$, and $0.38 \%$ of the phenotypic variation, respectively. Noticeably, a residual phenotypic variation of $14.59 \%$ (8.46 to $20.35 \%$ ) due to undetected QTLs and environment was observed.

Genetic stocks with improved blast resistance tagged with SNPs. A total of 30 new genetic stocks carrying a different combination of major blast $R$ genes, $P i-t a(q B R 12)$ and $P i-b(q B R 2)$, and minor QTLs $q B R 8, q B R 9$, and $q B R 3$ were resistant to all tested isolates except for TM2 (Table 4). Marker heterozygosity remaining in RIL26 at $q B R 9$, RIL116 at $P i$-ta, RIL183 at $q B R 8$, and RIL194 at $P i$-t $a$ are still suggests that these genetic stocks can also be used for fine mapping of these resistance QTLs. These 30 CSRILs with their disease reaction and nearest marker for resistance QTLs should be useful for breeding for improved resistance via a marker-assisted selection (MAS) approach.

\section{DISCUSSION}

A dozen new rice varieties with either the $P i$-ta gene cluster or $P i-b$ has been released in the two most recent decades in the United States, some of which are still being grown commercially, while others have been used as breeding parents (McClung et al. 2004; RoyChowdhury et al. 2012; Wang et al. 2008, 2010, 2015). In the present study, we first demonstrated that $P i$-ta was effective in preventing the major blast races IB-1, IB-17, and IB-49, which is consistent with several previous studies (Jia 2009; Jia et al. 2012, 2016). Similarly, $P i-b$ was effective in preventing blast races IB-1, IB-17, and IE-1K, which is also consistent with previous studies (McClung et al. 2004; RoyChowdhury et al. 2012; Vasudevan et al. 2016). Then we showed an additive-by-additive epistatic interaction of $P i-t a$ and $P i-b$ in the CS-RIL population with three minor QTLs to $M$. oryzae. We suggest that it is beneficial to stack both $P i-t a$ and $P i-b$ along with minor QTLs to improve blast resistance. Similarly, previous studies also demonstrated that enhanced resistance to $M$. oryzae can be achieved with more than one blast $R$ gene (Chen et al. 2008; Hittalmani et al. 2000; Liu et al. 2007; Ratna Madhavi et al. 2016).

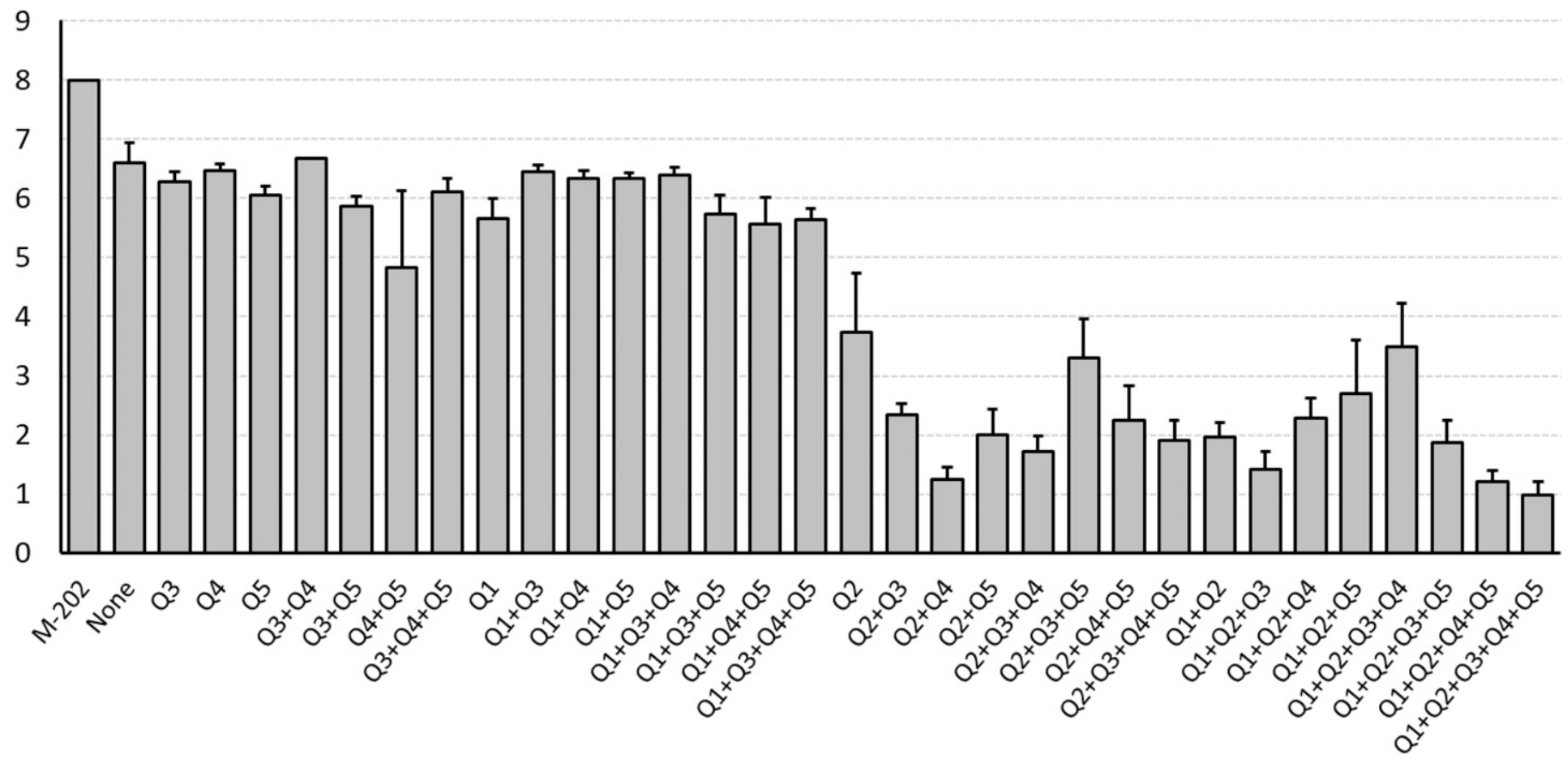

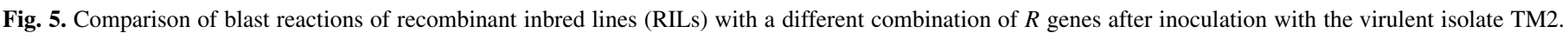

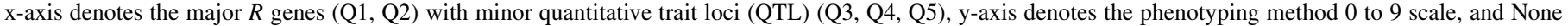
denotes RILs without any QTL. 
Major $R$ genes are not always effective for field resistance. As an alternative, minor resistance QTLs may be pyramided to achieve more effective resistance. In the present study, three minor resistance QTLs were identified in regions already reported by others to be associated with blast resistance in different mapping populations with different isolates under different environments. Namely, Q3 ( $q B R 8)$ and Q4 ( $q B R$ 9) were mapped in the same region as $q B L R 8$ and $q B L A S T 9.3$, respectively, with RILs of the cross of Lemont with Jasmine 85 (Jia and Liu 2011; Xing et al. 2015). Q5 $(q B R 3)$, detected with race IE-1K (TM2), was at the location of $q B R-Z F 1$ (Wu et al. 2016). Taken together, these findings suggest that minor resistance QTLs, $q B R 3, q B R 8$, and $q B R 9$, in rice have been used in breeding programs for preventing rice blast disease.

The monogenic lines with $P i$-ta and/or with $P i-b$ and digenic lines of lines with both $P i-t a$ and $P i-b$ in the same genetic background would be ideal materials for studying interactions of these two major $R$ genes. In the absence of such genetic materials, we detected precise phenotypic effects of $P i$-ta and $P i-b$ and other minor resistance QTLs with genotypic and phenotypic data of a recombinant inbred mapping population. QTLs were detected by screening the entire genome with Haley-Knott (HK) regression method to find the QTL with largest LOD, which was then fixed as an additive covariate before screening the genome again until no new QTLs over the threshold were identified. Using this method minor QTLs were identified without confounding effects by major QTLs. The QTLs effects were evaluated using statistical formula $\mathrm{y} \sim \mathrm{Q} 1+\mathrm{Q} 2+\ldots+\mathrm{Q} 1 * \mathrm{Q} 2$ to estimate the effect of single, pairwise QTL (Broman and Sen 2009). Despite the R/qtl analysis cannot detect common QTLs of both parents it was effective to identify different QTLs between two parents through regression interval mapping (RIM) with $1 \mathrm{cM}$ (Haley and Knott 1992) and was scanned by multiple interval mapping (MIM) (Kao et al. 1999). R/qtl was originally developed for $\mathrm{F}_{2}$ of mice and can be modified to analyze RIL data except marker alleles that are heterozygotes. In this case, the heterozygotes were treated as missing data.

Classical mapping studies with qualitative data have mapped over 100 major blast $R$ genes and 30 of them have been cloned (Wang et al. $2013,2017 \mathrm{a})$. In the present study, the categorical data were used to predict the precise phenotypic effect of major and minor $R$ genes. We evaluated the disease reaction of the CS-RIL population with the standard visual scale ( 0 to 9 ) developed by the International Rice Research Institute (IRRI 2002). We successfully detected two major QTLs, Q1 (Pi-ta) and Q2 (Pi-b), and three minor QTLs with 10 selected isolates. We also found that $14.59 \%$ of phenotypic variation was not associated with any genomic region. These phenotypic variations may be due to the variation of temperature and humidity under greenhouse conditions. The existences of these unaccounted phenotypic variations are consistent with the fact that variation of the temperature and humidity impacts on the pathogenicity of $M$. oryzae (Castejón-Muñoz 2008). Besides environmental effect, another possibility is that we failed to detect some resistance QTLs due to the limited density of genetic markers and number of the RILs.

In the past two decades, research has focused on dissecting single $R$ gene-mediated signal recognition and transduction in plant immunity. The mechanisms of disease resistance are now understood as two tiers of defense responses. The first tier of defense is PAMP-triggered immunity (PTI) which is involved in pathogen molecule recognition by plant extracellular receptors; and the second tier of defense is effector triggered immunity (ETI) which is the function of major $R$ genes, such as $P i$-ta or $P i-b$ (Dangl et al. 2013). For the first time, an accelerated defense response was detected in rice seedlings when both $P i-t a$ and $P i-b$ were stacked together in a large number of progeny of parents carrying each of them. Previously, Xiao et al. (2016) showed that a near-isogenic rice

TABLE 4. Improved genetic stocks with their disease reaction, nearest markers, and resistance quantitative trait loci (QTLs) ${ }^{\mathrm{x}}$

\begin{tabular}{|c|c|c|c|c|c|c|c|c|c|c|c|c|c|c|c|}
\hline \multirow[b]{2}{*}{2013 Plot } & \multirow[b]{2}{*}{ RIL } & \multicolumn{3}{|c|}{ IB1 } & \multicolumn{3}{|c|}{ IB17 } & \multicolumn{3}{|c|}{ IB49 } & \multirow{2}{*}{$\frac{\mathrm{Q} 1(P i-t a)}{\mathrm{id12004228}}$} & \multirow{2}{*}{$\frac{\mathrm{Q} 2(P i-b)}{\mathrm{id} 2015934}$} & \multirow{2}{*}{$\frac{\mathrm{Q} 3(q B R 8)}{\mathrm{rd} 8002343}$} & \multirow{2}{*}{$\frac{\mathrm{Q} 4(q B R 9)}{\mathrm{id} 9007173}$} & \multirow{2}{*}{$\frac{\mathrm{Q} 5(q B R 3)}{\operatorname{id} 3013770}$} \\
\hline & & $12 \mathrm{~A} 23$ & 15A6_3 & 15L22 & $14 \mathrm{~L} 42$ & 88A110 & SSID10 & ZN57 & ZN61 & $\mathrm{ML1}^{\mathrm{y}}$ & & & & & \\
\hline 13B1902 & 3 & 0.33 & 0.00 & 0.33 & 0.33 & 0.00 & 0.20 & 0.25 & 0.00 & 0.00 & + & + & - & - & - \\
\hline 13B 1924 & 26 & 1.00 & 0.00 & 0.00 & 0.00 & 0.00 & 0.20 & 0.00 & 0.00 & 0.25 & + & + & + & $\mathrm{H}^{\mathrm{z}}$ & + \\
\hline 13B1925 & 27 & 0.50 & 0.67 & 0.00 & 0.33 & 0.17 & 0.20 & 0.00 & 0.20 & 0.00 & + & + & + & + & - \\
\hline 13B1934 & 36 & 0.00 & 0.00 & 0.00 & 0.67 & 0.00 & 0.20 & 0.00 & 0.00 & 0.25 & + & + & - & + & + \\
\hline 13B1939 & 41 & 0.33 & 0.33 & 0.00 & 0.00 & 0.17 & 0.40 & 0.25 & 0.00 & 0.25 & + & + & + & - & - \\
\hline 13B1943 & 45 & 0.50 & 0.00 & 0.33 & 0.00 & 0.33 & 0.50 & 0.00 & 0.00 & 0.00 & + & + & + & - & - \\
\hline 13B1955 & 59 & 0.00 & 0.00 & 0.00 & 0.33 & 0.00 & 0.20 & 0.00 & 0.40 & 0.00 & + & + & + & - & + \\
\hline 13B1980 & 86 & 1.33 & 0.00 & 0.00 & 0.33 & 0.00 & 0.00 & 0.25 & 0.00 & 0.00 & + & + & - & - & + \\
\hline $13 \mathrm{~B} 2008$ & 116 & 0.33 & 0.00 & 0.00 & 0.67 & 0.00 & 0.40 & 0.00 & 0.00 & 0.00 & $\mathrm{H}$ & + & - & + & + \\
\hline $13 B 2022$ & 128 & 2.00 & 0.00 & 0.00 & 0.00 & 0.00 & 0.00 & 0.25 & 0.00 & 0.00 & + & + & + & - & + \\
\hline $13 B 2029$ & 135 & 0.00 & 0.00 & 0.00 & 0.00 & 0.00 & 0.20 & 0.00 & 0.40 & 0.00 & + & + & + & - & - \\
\hline 13B2045 & 150 & 0.33 & 0.00 & 0.00 & 0.67 & 0.00 & 0.40 & 0.00 & 0.00 & 0.00 & + & + & + & + & + \\
\hline 13B2060 & 166 & 0.50 & 0.00 & 0.00 & 0.33 & 0.00 & 0.20 & 0.00 & 0.00 & 0.00 & + & + & - & + & - \\
\hline 13B2063 & 169 & 0.33 & 0.00 & 0.00 & 0.67 & 0.00 & 0.00 & 0.25 & 0.00 & 0.25 & + & + & - & - & + \\
\hline 13B2078 & 183 & 0.00 & 0.00 & 0.33 & 1.00 & 0.40 & 0.40 & 0.00 & 0.00 & 0.00 & + & + & $\mathrm{H}$ & + & + \\
\hline 13B2089 & 194 & 0.00 & 0.00 & 0.33 & 0.00 & 0.00 & 1.00 & 0.00 & 0.00 & 0.00 & $\mathrm{H}$ & + & - & + & + \\
\hline 13B2091 & 197 & 0.33 & 0.00 & 0.00 & 0.00 & 0.33 & 0.40 & 0.00 & 0.00 & 0.25 & + & + & - & - & - \\
\hline $13 \mathrm{~B} 2098$ & 204 & 1.33 & 0.00 & 0.00 & 0.67 & 0.00 & 0.20 & 0.00 & 0.00 & 0.00 & + & + & + & + & + \\
\hline $13 \mathrm{~B} 2102$ & 208 & 0.00 & 0.00 & 0.00 & 0.33 & 0.17 & 0.60 & 0.00 & 0.00 & 0.00 & + & + & - & + & + \\
\hline 13B2107 & 213 & 0.67 & 0.00 & 0.33 & 0.00 & 0.17 & 0.00 & 0.50 & 0.20 & 0.25 & + & - & + & + & + \\
\hline 13B2114 & 221 & 0.67 & 0.00 & 0.33 & 0.67 & 0.17 & 0.20 & 0.00 & 0.20 & 0.00 & + & + & + & - & + \\
\hline 13B2115 & 222 & 0.33 & 0.33 & 0.00 & 0.00 & 0.00 & 0.40 & 0.25 & 0.20 & 0.75 & + & - & + & + & + \\
\hline 13B2124 & 231 & 0.00 & 0.00 & 0.33 & 0.00 & 0.00 & 0.00 & 0.25 & 0.00 & 0.50 & + & + & - & - & - \\
\hline 13B2129 & 236 & 0.33 & 0.00 & 1.00 & 0.00 & 0.17 & 0.00 & 0.50 & 0.00 & 0.00 & - & + & - & + & + \\
\hline 13B2136 & 243 & 0.00 & 0.00 & 0.00 & 0.67 & 0.17 & 0.00 & 0.00 & 0.00 & 0.00 & + & + & - & + & + \\
\hline 13B2137 & 244 & 0.00 & 0.00 & 0.00 & 1.00 & 0.17 & 0.50 & 0.00 & 0.00 & 0.00 & + & + & - & + & + \\
\hline $13 \mathrm{~B} 2139$ & 246 & 0.00 & 0.00 & 0.00 & 1.00 & 0.60 & 0.00 & 0.00 & 0.00 & 0.00 & + & + & + & + & + \\
\hline $13 B 2144$ & 251 & 0.33 & 0.00 & 0.00 & 0.00 & 0.00 & 0.40 & 0.50 & 0.00 & 0.25 & + & + & - & + & - \\
\hline $13 \mathrm{~B} 2146$ & 253 & 0.33 & 0.00 & 0.00 & 1.00 & 0.75 & 0.20 & 0.00 & 0.00 & 0.00 & + & + & - & + & + \\
\hline 13B2163 & 271 & 0.33 & 0.00 & 0.00 & 0.33 & 0.33 & 0.00 & 0.00 & 0.40 & 0.00 & + & + & + & + & + \\
\hline
\end{tabular}

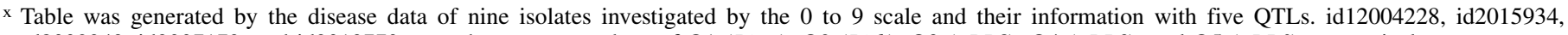
rd8002343, id9007173, and id3013770 were the nearest markers of Q1 (Pi-ta), Q2 (Pi-b), Q3 ( $q B R 8)$, Q4 ( $q B R 9)$, and Q5 ( $q B R 3)$, respectively.

y ML1 indicates an isolate purified from race IB-49.

${ }^{\mathrm{z}} \mathrm{H}$ denotes heterozygous. 
line (NIL) with $P i-46(t)$ and $P i$-ta was resistant to panicle blast whereas NILs either with $P i$-ta or $P i-46(t)$ were not. Taken together, we suggest that there is a sophisticated genetic control mechanism that exists during the resistant response to $M$. oryzae mediated by major blast $R$ genes.

The role of $P i$-ta is to trigger ETI when plants are challenged with avirulent pathogens with AVR-Pital (Bryan et al. 2000). In the absence of avirulent pathogens, Pi-ta presented no effect on disease reactions of CS-RILs with different combinations of minor QTLs. In contrast, enhanced resistance was observed when $P i$ - $b$ was stacked with different minor QTLs. Similarly, we detected small positive additive effects of RILs with $P i$ - $t a$ and Q4 or RILs with $P i$ - $b$ and Q4 with the isolate 15L22. These findings suggest that major $R$ gene-triggered immunity may enhance the defense response mediated by PTI. Cloning of minor QTLs will help to understand how minor QTLs identified in this study are responsible for the observed PTI.

In summary, understanding the genetic basis of the interaction between major $R$ genes and minor QTLs is essential for ensuring the effectiveness of their utilization for improving blast resistance. We showed that enhanced blast resistance can be achieved by a genetic combination of $P i-t a, P i-b$, and minor QTLs in CS-RILs. Further validation with rice lines of gene combination in the same genetic background will be required. Nevertheless, this knowledge not only is important for stacking both $P i-t a, P i-b$, and minor QTLs to develop blast-resistant rice varieties worldwide, but also for investigating the molecular basis of $R$ gene-mediated signaling recognition and transduction pathway. Minor resistance QTLs $q B R 3$, $q B R 8$, and $q B R 9$ have been widely utilized in breeding programs in different parts of the world. Utilization of these resistance QTLs can extend the effectiveness of major blast $R$ genes. Thirty resistant long-grain genetic stocks tagged with SNP (Table 4) can be used as blast-resistant donors for MAS and four segregating RILs are ideal genetic materials for fine mapping and cloning of blast resistance QTLs.

\section{ACKNOWLEDGMENTS}

We thank the China Scholarship Council (CSC) for supporting X. Chen's living and traveling expenses in the United States; D. Wu of Taiwan Agricultural Research Institute for excellent assistance on the data analysis; H. Zhao for his advice on QTLs analysis; and T. Bianco of the U.S. Department of Agriculture-Agricultural Research Service for manuscript proofreading and fungal culture assistance. The USDA is an equal opportunity provider and employer.

\section{LITERATURE CITED}

Ballini, E., Morel, J.-B., Droc, G., Price, A., Courtois, B., Notteghem, J.-L., and Tharreau, D. 2008. A genome-wide meta-analysis of rice blast resistance genes and quantitative trait loci provides new insights into partial and complete resistance. Mol. Plant-Microbe Interact. 21:859-868.

Broman, K. W., and Sen, Ś. 2009. A Guide to QTL Mapping with R/QTL. Springer, New York.

Bryan, G. T., Wu, K.-S., Farrall, L., Jia, Y., Hershey, H. P., McAdams, S. A., Faulk, K. N., Donaldson, G. K., Tarchini, R., and Valent, B. 2000. A single amino acid difference distinguishes resistant and susceptible alleles of the rice blast gene Pi-ta. Plant Cell 12:2033-2045.

Carlborg, Ö., and Haley, C. S. 2004. Epistasis: Too often neglected in complex trait studies? Nat. Rev. Genet. 5:618-625.

Castejón-Muñoz, M. 2008. The effect of temperature and relative humidity on the airborne concentration of Pyricularia oryzae spores and the development of rice blast in southern Spain. Span. J. Agric. Res. 6:61-69.

Chen, H., Chen, Z., Ni, S., Zuo, S., Pan, X., and Zhu, X. 2008. Pyramiding three genes with resistance to blast by marker-assisted selection to improve rice blast resistance of Jin 23B. Chin. J. Rice Sci. 22:23-27.

Churchill, G. A., and Doerge, R. W. 1994. Empirical threshold values for quantitative trait mapping. Genetics 138:963-971.

Correll, J. C., Harp, T. L., Guerber, J. C., Zeigler, R. S., Liu, B., Cartwright, R. D., and Lee, F. N. 2000. Characterization of Pyricularia grisea in the United States using independent genetic and molecular markers. Phytopathology 90:1396-1404.
Counce, P. A., Keisling, T. C., and Mitchell, A. J. 2000. A uniform, objective, and adaptive system for expressing rice development. Crop Sci. 40: 436-443.

Dangl, J. L., Horvath, D. M., and Staskawicz, B. J. 2013. Pivoting the plant immune system from dissection to deployment. Science 341:746-751.

Fjellstrom, R. G., Conaway-Bormans, C. A., McClung, A. M., Marchetti, M. A., Shank, A. R., and Park, W. D. 2004. Development of DNA markers suitable for marker assisted selection of three $P i$ genes conferring resistance to multiple Pyricularia grisea pathotypes. Crop Sci. 44:1790-1798.

Fukuoka, S., Saka, N., Mizukami, Y., Koga, H., Yamanouchi, U., Yoshioka, Y., Hayashi, N., Ebana, K., Mizobuchi, R., and Yano, M. 2015. Gene pyramiding enhances durable blast disease resistance in rice. Sci. Rep. 5:7773.

Gibbons, J. W., Moldenhauer, K. A. K., Gravois, K., Lee, F. N., Bernhardt, J. L., Meullenet, J.-F., Bryant, R., Anders, M., Norman, R. J., Cartwright, R., Taylor, K., Bulloch, J., and Blocker, M. M. 2006. Registration of 'Cybonnet' rice. Crop Sci. 46:2317-2318.

Haley, C. S., and Knott, S. A. 1992. A simple regression method for mapping quantitative trait loci in line crosses using flanking markers. Heredity $69: 315-324$.

Hittalmani, S., Parco, A., Mew, T. V., Zeigler, R. S., and Huang, N. 2000. Fine mapping and DNA marker-assisted pyramiding of the three major genes for blast resistance in rice. Theor. Appl. Genet. 100:1121-1128.

International Rice Research Institute (IRRI). 2002. Standard evaluation system for rice. IRRI, Manila, the Philippines.

Jia, Y. 2009. Artificial introgression of a large chromosome fragment around the rice blast resistance gene $P$-ta in backcross progeny and several elite rice cultivars. Heredity 103:333-339.

Jia, Y., Bryan, G. T., Farrall, L., and Valent, B. 2003a. Natural variation at the $P i$-ta rice blast resistance locus. Phytopathology 93:1452-1459.

Jia, Y., Jia, M. H., Wang, X., and Liu, G. 2012. Indica and japonica crosses resulting in linkage block and recombination suppression on rice chromosome 12. PLoS One 7:e43066.

Jia, Y., and Liu, G. 2011. Mapping quantitative trait loci for resistance to rice blast. Phytopathology 101:176-181.

Jia, Y., McAdams, S. A., Bryan, G. T., Hershey, H. P., and Valent, B. 2000. Direct interaction of resistance gene and avirulence gene products confers rice blast resistance. EMBO J. 19:4004-4014.

Jia, Y., Redus, M., Wang, Z., and Rutger, J. N. 2004a. Development of a SNLP marker from the $P i$-ta blast resistance gene by tri-primer PCR. Euphytica 138:97-105.

Jia, Y., Valent, B., and Lee, F. N. 2003b. Determination of host responses to Magnaporthe grisea on detached rice leaves using a spot inoculation method. Plant Dis. 87:129-133.

Jia, Y., Wang, Z., Fjellstrom, R. G., Moldenhauer, K. A. K., Azam, M. A., Correll, J., Lee, F. N., Xia, Y., and Rutger, J. N. 2004b. Rice Pi-ta gene confers resistance to the major pathotypes of the rice blast fungus in the United States. Phytopathology 94:296-301.

Jia, Y., Wang, Z., and Singh, P. 2002. Development of dominant rice blast Pi-ta resistance gene markers. Crop Sci. 42:2145-2149.

Jia, Y., Zhou, E., Lee, S., and Bianco, T. 2016. Co-evolutionary dynamics of rice blast resistance gene $\mathrm{Pi}$-ta and Magnaporthe oryzae avirulence gene AVR-Pital. Phytopathology 106:676-683.

Kao, C. H., Zeng, Z. B., and Teasdale, R. D. 1999. Multiple interval mapping for quantitative trait loci. Genetics 152:1203-1216.

Karamian, F., Heydari Nezhad, A. M., Nokhbeh Zaeim, A., Moradi, K., and Drakhshan, A. H. 2015. Blast disease in rice: a review. IJSRST 5:228-232.

Kosambi, D. D. 1943. The estimate of map distances from recombination values. Ann. Eugen. 12:172-175.

Lee, S., Costanzo, S., Jia, Y., Olsen, K., and Caicedo, A. 2009. Evolutionary dynamics of the genomic region around the blast resistance gene Pi-ta in AA genome Oryza species. Genetics 183:1315-1325.

Liu, X., Zhao, M., He, Y., Chen, D., Xiao, Y., and Lei, C. 2007. Breeding and blast resistance identification of Lijiangxintuanheigu near-isogenic pyramid lines. Acta Agron. Sin. 33:20-24.

Liu, Y., Qi, X., Gealy, D. R., Olsen, K. M., Caicedo, A. L., and Jia, Y. 2015a. QTL analysis for resistance to blast disease in U.S. weedy rice. Mol. PlantMicrobe Interact. 28:834-844.

Liu, Y., Qi, X., Young, N. D., Olsen, K. M., Caicedo, A. L., and Jia, Y. 2015 b. Characterization of resistance genes to rice blast fungus Magnaporthe oryzae in a "Green Revolution" rice variety. Mol. Breed. 35:52.

Ma, J., Jia, M. H., and Jia, Y. 2014. Characterization of rice blast resistance gene Pi61( $t)$ in rice germplasm. Plant Dis. 98:1200-1204.

McClung, A. M., Fjellstrom, R. G., Bergman, C. J., Bormans, C. A., Park, W. D., and Marchetti, M. A. 2004. Registration of 'Saber' rice. Crop Sci. 44:693-694.

Nalley, L., Tsiboe, F., Durand-Morat, A., Shew, A., and Thoma, G. 2016. Economic and environmental impact of rice blast pathogen (Magnaporthe oryzae) alleviation in the United States. PLoS One 11:e0167294.

Pennisi, E. 2010. Armed and dangerous. Science 327:804-805. 
Phillips, P. C. 2008. Epistasis-the essential role of gene interaction in the structure and evolution of genetic systems. Nat. Rev. Genet. 9:855-867.

Pinson, S. R. M., Jia, Y., and Gibbons, J. W. 2013. Three quantitative trait loci conferring resistance to kernel fissuring in rice identified by selective genotyping in two tropical japonica populations. Crop Sci. 53:2434-2443.

R Core Team. 2013. R: A language and environment for statistical computing. R Core Team. http://www.R-project.org

Ratna Madhavi, K., Rambabu, R., Abhilash Kumar, V., Vijay Kumar, S., Aruna, J., Ramesh, S., Sundaram, R. M., Laha, G. S., Sheshu Madhav, M., Ravindra Babu, V., and Srinivas Prasad, M. 2016. Marker assisted introgression of blast ( $\mathrm{Pi}-2$ and $\mathrm{Pi}-54$ ) genes in to the genetic background of elite, bacterial blight resistant indica rice variety, improved Samba Mahsuri. Euphytica 212:331-342.

RoyChowdhury, M., Jia, Y., Jia, M. H., Fjellstrom, B., and Cartwright, R. 2012. Identification of the rice blast resistance gene $P i-b$ in the national small grains collection. Phytopathology 102:700-706.

Sen, S., and Churchill, G. A. 2001. A statistical framework for quantitative trait mapping. Genetics 159:371-387.

Sharma, T. R., Rai, A. K., Gupta, S. K., Vijayan, J., Devanna, B. N., and Ray, S. 2012. Rice blast management through host plant resistance: Retrospect and prospects. Agric. Res. 1:37-52.

Silué, D., Nottenghem, J.-L., and Tharreau, D. 1992. Evidence for a gene-forgene relationship in the Oryza sativa-Magnaporthe grisea pathosystem. Phytopathology 82:577-580.

Thomson, M. J., Zhao, K., Wright, M., McNally, K. L., Rey, J., Tung, C.-W., Reynolds, A., Scheffler, B. E., Eizenga, G. C., McClung, A. M., Kim, H., Ismail, A. M., de Ocampo, M., Mojica, C., Reveche, M. Y., Dilla-Ermita, C. J., Mauleon, R., Leung, H., Bustamante, C., and McCouch, S. R. 2012. High-throughput single nucleotide polymorphism genotyping for breeding applications in rice using the BeadXpress platform. Mol. 29:875-886.

Vasudevan, K., Vera Cruz, C. M., Gruissem, W., and Bhullar, N. K. 2016. Geographically distinct and domain-specific sequence variations in the alleles of rice blast resistance gene Pib. Front. Plant Sci. 7:915.

Voorrips, R. E. 2002. MapChart: Software for the graphical presentation of linkage maps and QTL. J. Hered. 93:77-78.

Wang, B. H., Ebbole, D. J., and Wang, Z. H. 2017a. The arms race between Magnaporthe oryzae and rice: Diversity and interaction of $A v r$ and $R$ genes. J. Integr. Agric. 16:2746-2760.
Wang, X., Fjellstrom, R., Jia, Y., Yan, W. G., Jia, M. H., Scheffler, B. E., Wu, D., Shu, Q., and McClung, A. 2010. Characterization of Pi-ta blast resistance gene in an international rice core collection. Plant Breed. 129:491-501.

Wang, X., Jia, M. H., Ghai, P., Lee, F. N., and Jia, Y. 2015. Genome-wide association of rice blast resistance and yield related components of rice. Mol. Plant-Microbe Interact. 28:1383-1392.

Wang, X., Jia, Y., Shu, Q., and Wu, D. 2008. Haplotype diversity at the Pi-ta locus in cultivated rice and its wild relatives. Phytopathology 98:1305-1311.

Wang, X., Jia, Y., Wamishe, Y., Jia, M. H., and Valent, B. 2017b. Dynamic changes in the rice blast population in the United States over six decades. Mol. Plant-Microbe Interact. 30:803-812.

Wang, X., Lee, S., Wang, J., Ma, J., Bianco, T., and Jia, Y. 2013. Current advances on genetic resistance to rice blast disease. Pages 195-217 in: RiceGermplasm, Gene and Improvement. W. Yan and J. Bao, eds. InTech, Rijeka, Croatia.

Wang, Z.-X., Yamanouchi, U., Katayose, Y., Sasaki, T., and Yano, M. 2001. Expression of the $P i b$ rice-blast-resistance gene family is up-regulated by environmental conditions favoring infection and by chemical signals that trigger secondary plant defenses. Plant Mol. Biol. 47:653-661.

Wang, Z.-X., Yano, M., Yamanouchi, U., Iwamoto, M., Monna, L., Hayasaka, H., Katayose, Y., and Sasaki, T. 1999. The Pib gene for rice blast resistance belongs to the nucleotide binding and leucine-rich repeat class of plant disease resistance genes. Plant J. 19:55-64.

Wu, Y. Y., He, J. B., Li, A. H., Fang, N. Y., He, W. W., Dang, L. L., Zeng, G. Y., Huang, J., Bao Y. M., and Zhang, H. S. 2016. Population structure analysis and association mapping of blast resistance in indica rice (Oryza sativa L.) landraces. Genet. Mol. Res. 15.

Xiao, W.-M., Luo, L.-X., Wang, H., Guo, T., Liu, Y.-Z., Zhou, J.-Y., Zhu, X.-Y., Wang, Q.-Y., and Chen, Z.-Q. 2016. Pyramiding of Pi46 and Pi-ta to improve blast resistance and to evaluate the resistance effect of the two $R$ genes. J. Integr. Agric. 15:2290-2298.

Xing, J., Jia, M. H., Correll, J. C., Yuan, L., Deng, H., and Jia, Y. 2015. Confirming and identifying new loci for rice blast disease resistance using Magnaporthe oryzae field isolates in the U.S. Crop Sci. 55:2620-2627.

Yang, H., Jia, M. H., Jia, Y., Xing, J., Venu, R.-C., Bellizzi, M., Yuan, L., Wang, Z., Sun, C., and Wang, G.-L. 2013. Molecular mapping of four blast resistance genes using recombinant inbred lines of 93-11 and Nipponbare. J. Plant Biol. 56:91-97. 